\title{
Developing Training Applications for Hydrogen Emergency Response Training
}

\author{
Darque Pinto \\ UTAD \\ Vila Real, Portugal \\ darquepinto@gmail.com
}

\author{
Miguel Melo \\ INESC TEC \\ Porto, Portugal \\ mcmelo@inesctec.pt
}

\author{
Bruno Peixoto \\ UTAD \\ Vila Real, Portugal \\ brunomepeixoto@gmail.com
}

\author{
Vasco Amorim \\ UTAD and INESC TEC \\ Vila Real and Porto, Portugal \\ eamorim@utad.pt
}

\author{
Guilherme Gonçalves \\ UTAD \\ Vila Real, Portugal \\ guilhermeg@utad.pt
}

\author{
Maximino Bessa \\ UTAD and INESC TEC \\ Vila Real and Porto, Portugal \\ maxbessa@utad.pt
}

\begin{abstract}
Virtual Reality (VR) has been evolving over the years, becoming more and more accessible, in a wide area of applications. One of these areas where VR can have a major impact is training and certification. Hydrogen vehicles are becoming a reality and first responders still lack proper tools and resources to train emergency responses for the purpose. VR can play here a crucial role in ensuring a proper hydrogen emergency response training due to the advantages associated with VR training programs such as resource optimization, repeatability, and replicability.

This paper proposes using VR for hydrogen emergency response training by developing a solution composed of three components: tutorial mode, training mode, and certification mode. A usability study is further conducted to evaluate its usability and user satisfaction. The results show that the use of this application regards usability and user satisfaction were extremely positive.
\end{abstract}

Index Terms-Virtual Reality, Usability, User Satisfaction, Training

\section{INTRODUÇÃO}

A Realidade Virtual (RV) teve um acréscimo em termos de acessibilidade tecnológica, os equipamentos ficaram mais alcançáveis pelo público interessado. Com isto, também aumentou o financiamento de entidades para o desenvolvimento direcionado a esta tecnologia. Tendo igualmente em conta o aumento da qualidade dos ambientes virtuais é então possível a entrega de uma simulação completamente imersiva e realista.

Recentemente a RV tem recebido muita atenção em diversas áreas devido ao potencial que representa. Esta permite criar uma simulação da realidade capaz de envolver o utilizador de tal forma que o ambiente virtual passa a ser a sua nova realidade, permitindo que a RV seja usada para treinar profissionais a baixo custo e com maior segurança.

$\mathrm{O}$ realismo num ambiente virtual tem um papel significativo aquando relacionado com o treino de situações arriscadas e que necessitam de alguma meticulosidade [1], tendo em conta

978-1-7281-6378-9/19/\$31.00 @2019 IEEE que simulações que são o mais próximo possível da realidade providenciam imersividade ao utilizador, implicando assim que este interaja com o ambiente de forma natural, tal como o faria na realidade [2].

Para que o treino em RV seja eficaz em replicar as condições reais, é necessário perceber se a interação para com os objetos virtuais é fiel e fácil de entender. Se, por exemplo, um simulador virtual que pretende treinar cirurgiões não replicar fielmente o comportamento dos utensílios médicos além de ser complexo o seu manuseamento através da RV poderá resultar em uso indevido destes mesmos instrumentos na realidade. É de notar que, por vezes, não é possível replicar o manuseamento de instrumentos/utensílios devido a limitações técnicas dos comandos virtuais e/ou falta de feedback háptico e/ou a extensão e fidelidade do tracking do corpo humano. Neste caso onde não é possível criar uma réplica fiel do manuseamento dos instrumentos, será importante criar uma metáfora de interação, o mais semelhante à realidade dentro dos possíveis, mas adaptada para ser usada em RV. Será importante igualmente que os profissionais entendam como os instrumentos serão manuseados num caso real.

As equipas de bombeiros todos os dias se deparam com situações de risco onde decisões têm que ser tomadas com o mais breve tempo possível. De modo que estas decisões sejam corretamente aplicadas, é necessário treino prévio a baixo custo monetário e de completa segurança, tendo em conta que se tratam de situações de risco reais onde seja possível treinar as aptidões dos indivíduos. Com uma simulação em RV é possível treinar vezes sem conta (repetível), em completa segurança, com controlo sobre todas as variáveis e a baixo custo além de ser replicável.

Neste caso de estudo, é recriada uma situação de emergência que engloba um incêndio num veículo alimentado a hidrogénio. Estes veículos contêm tanques de hidrogénio sob pressão. Durante um incêndio, estes tanques vão aquecendo e caso não sejam arrefecidos poderão ultrapassar o limite de 
pressão máxima. Caso chegue aos $110^{\circ} \mathrm{C}$, uma válvula de segurança irá abrir e libertar o gás de maneira a evitar risco de explosão, sendo queimado à medida que sai do tanque. Duas propriedades que fazem deste gás perigoso é o fato de ser incolor e inodoro. A chama de hidrogénio torna-se invisível, sendo possível apenas visualizar a distorção criada pelo calor e ouvir o som do gás a ser libertado sob pressão, sendo um dos indicadores iniciais de que existe uma fuga. Esta válvula assim que abre não volta a fechar, sendo necessário esperar até que todo o hidrogénio seja queimado. Face a estas circunstâncias, os bombeiros têm um conjunto de procedimentos a realizar dependendo da situação em que se encontra a fuga.

Este estudo, direcionado para o treino e certificação de bombeiros, tem como objetivo verificar a usabilidade $\mathrm{e}$ satisfação de uma aplicação de RV que permite treinar a resposta a situações de perigo, mais especificamente situações que envolvam a libertação de hidrogénio em veículos ligeiros.

\section{ESTADO DA ARTE}

Segundo Tuli Sulbaran e Nelson C. Baker [3] a maior vantagem da Realidade Virtual (RV) é a informação visual e a interatividade, e toda a retenção de conhecimento depende dessa vantagem para atingir sucesso. Os autores mostraram também que os utilizadores normalmente tendem a gostar mais do treino em ambientes virtuais, quando comparado com métodos tradicionais, retendo mais conhecimento. $\mathrm{O}$ estudo de Shana Smith e Emily Ericson [4] vem a salientar o que antes foi referido através de uma simulação que teve como objetivo treinar crianças para situações de incêndio e como deveriam proceder em segurança. Os resultados indicaram que a RV poderá perfeitamente criar um novo paradigma de aprendizagem, sendo possível a substituição dos métodos tradicionais, tendo em conta que os utilizadores demonstraram preferência por este tipo de treino/aprendizagem.

David L. Tate et al. [5] realizaram um estudo, com uma simulação do navio Shadwell, com bombeiros da marinha treinados. O objetivo do estudo era tentar perceber se o conhecimento prévio do ambiente do navio ajudaria na realização mais rápida da tarefa em mãos. Os resultados das experiências foram conclusivos, podendo assim afirmar que ambientes virtuais, para além de ajudarem a perceber como é o ambiente que ainda não conhecem, também ajudam efetivamente a praticar procedimentos com o fumo e incêndio simulado sem que seja necessário arriscar vidas e propriedades. Os autores confirmam assim que os resultados sugerem que treino num ambiente virtual pode contribuir para o aumento do desempenho dos bombeiros, reduzindo o tempo de demora para extinguir um incêndio.

No estudo experimental de L.Gamberini et al. [6], em que avaliaram as respostas dos participantes mediante a aparição inesperada de incêndio, foi possível aos autores a confirmação de que é válida a utilização de ambientes em RV tanto para pesquisa como para treino de estratégias necessárias em situações de emergência reais em espaços públicos. Foi-lhes possível sustentar esta afirmação tendo em conta que os par- ticipantes, do seu estudo, demonstraram respostas adaptativas às situações propostas.

Diversos autores tiveram como objetivo o desenvolvimento e implementação de ambientes em RV focados no treino e avaliação de bombeiros, dando foco ao desempenho individual [7] [2] [8] [9] e em equipa [10], de modo a melhorar e a treinar a capacidade destes profissionais de forma segura e repetitiva. Existem igualmente ambientes relacionados com simulações de desastres relacionados com incêndios, como, por exemplo o estudo de Aizhun Ren et al. [11], em que os resultados mostraram que o sistema construído em RV pode ser usado para evacuação e avaliação de segurança do edifício onde ocorre o incêndio, salientando assim o possível uso de RV para o treino relacionado com situações de perigo. Todo este desenvolvimento e investigação comprova que os ambientes virtuais são uma poderosa ferramenta para complementar o treino de bombeiros [12].

$\mathrm{Na}$ realização de uma aplicação de RV, especialmente no que diz respeito ao treino e certificação, a usabilidade é uma métrica fundamental para avaliar se o sistema é realmente utilizável. Para tal, existem diversos questionários que o permitem fazer [13], tais como o Questionnaire for User Interaction Satisfaction [14], o Software Usability Measurement Inventor [15], o System Usability Scale [16], ou o Usability Magnitude Estimation metric [17]. O questionário mais comum é o System Usability Scale (SUS) [18]. Este questionário é considerado ser uma ferramenta valiosa para ajudar a avaliar a qualidade da usabilidade de uma aplicação, permitindo avaliar se a aplicação tem problemas de usabilidade [13], [18]. No entanto, um dos lados negativos do SUS é o facto de este não avaliar a satisfação do utilizador; para tal existe o After-Scenario Questionnaire (ASQ) [19] que permite avaliar a satisfação do participante com a usabilidade do sistema, como a facilidade de conclusão da tarefa, o tempo de conclusão e adequação das informações de suporte.

\section{Metodologia}

Foi realizado um estudo descritivo com utilizadores de forma a avaliar a usabilidade e satisfação da aplicação. Dependendo dos dados obtidos foi igualmente realizado um estudo experimental.

\section{A. Amostra}

Esta experiência contou com a participação de 10 elementos (1 mulher e 9 homens) do ensino superior (estudantes e investigadores), com idades entre os 22 e os 31 anos $(M=24,9$, $\mathrm{DP} \approx 2,64)$. Todos estes relataram ter visão normal ou corrigida para o normal. Os participantes revelaram ter um bom conhecimento não só de computadores mas também acerca da RV. Relativamente a hábitos de jogo foi possível aferir que os participantes costumam jogar frequentemente. A participação foi totalmente voluntária e confidencial.

\section{B. Variáveis}

As variáveis dependentes consideradas foram a usabilidade do sistema, satisfação, os erros no procedimento (ambiente de 
treino, ambiente urbano, total). Estes erros podem ser de dois tipos: sair fora da área de segurança e/ou deixar os tanques de hidrogénio aquecerem além dos $110^{\circ} \mathrm{C}$. Caso o participante não cometa nenhum destes erros, é considerado que efetuou todo o procedimento corretamente para esse mesmo nível. Caso não tenha sido efetuado qualquer erro nestes dois níveis, considera-se que o participante não efetuou erros no total da experiência. Foram considerados igualmente a experiência prévia com RV, jogos e computadores.

\section{Materiais}

Foi utilizado um computador com processador Intel Core i76700K @ 4.00GHz e placa gráfica NVIDIA GeForce 1080 que era responsável não só por correr o motor de jogo, como todos os dispositivos de entrada e saída necessários para efetuar a experiência. No que diz respeito ao estímulo visual, os participantes utilizaram o HTC Vive HMD, que permite o tracking da posição da cabeça do participante, em todos os níveis. Este HMD possui um ângulo de visualização de 110 , uma resolução por olho de 1080x1200 pixels e uma taxa de atualização de $90 \mathrm{~Hz}$. Os comandos HTC VIVE permitiram o tracking das mãos e a possibilidade de interagir com o ambiente virtual. $\mathrm{O}$ estímulo de áudio foi entregue através de auscultadores com cancelamento ativo de ruído (Bose QuietComfort 25).

$\mathrm{O}$ estudo baseia-se num ambiente virtual que foi desenvolvido com recurso ao motor de jogo Unity (versão 2018.3.6f1). Este ambiente virtual está dividido em três partes, sendo a primeira um nível de tutorial, a segunda um nível de treino (com ajudas visuais) e a terceira um nível urbano (sem ajudas visuais). O nível de tutorial consiste numa sala que dá indicações (Fig. 1) ao participante de como utilizar os comandos para interagir e como se teletransportar, tal como indicar as ações necessárias para realizar o procedimento da experiência corretamente (Fig. 2). As instruções aparecem em texto e ilustrações e o utilizador terá de realizar um conjunto de pequenas tarefas onde aprende a interagir com o bocal da mangueira e com a locomoção no ambiente virtual. A interação com o bocal foi feita de maneira a que seja o mais natural possível e equivalente ao uso real. $\mathrm{O}$ bocal tem uma válvula que permite regular a intensidade do fluxo de água e um anel na extremidade que permite variar o ângulo com que esta sai do bocal. O utilizador segura a base da mangueira com uma das mãos enquanto que a outra controla a intensidade do fluxo ou o ângulo. Para controlar o fluxo de água o utilizador deve colocar o comando sobre a pega no topo do bucal e puxar para a frente ou para trás para controlar a intensidade. Para controlar o ângulo, o utilizador deve pegar o anel à frente do bucal e rodar para a esquerda ou direita de forma a controlar a abertura. A locomoção é realizada através de teletransporte, ao apontar o comando para o local onde o utilizador se pretende deslocar.

O nível de treino é uma replica de uma área de treino de um centro de formação e instrução de bombeiros, permite ao participante poder treinar os procedimentos necessários numa situação de incêndio envolvendo tanques de hidrogénio.

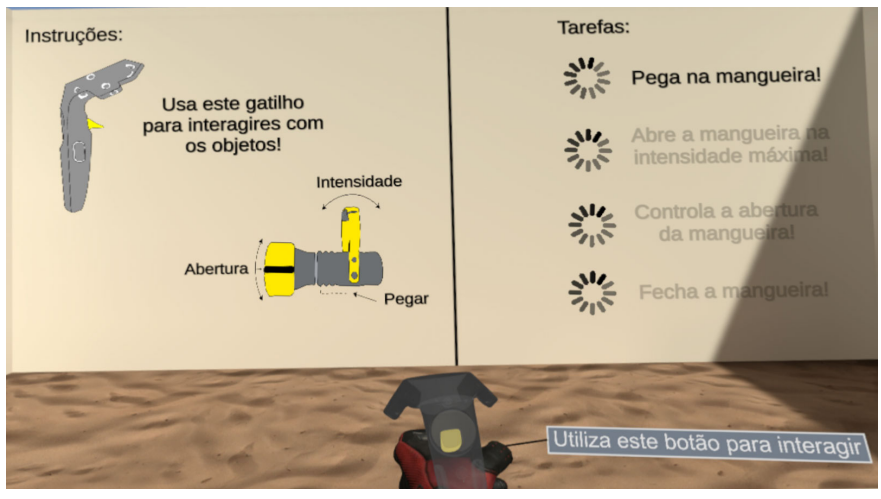

Fig. 1. Nível Tutorial com algumas indicações dirigidas ao participante.

Um dos primeiros objetivos é evitar que se acumule demasiada pressão nos tanques de hidrogénio que leve a válvula de segurança a abrir. Se tal acontecer o gás será libertado e queimado à medida que sai, mas a válvula não volta a fechar. Os bombeiros terão então de esperar que todo o gás seja queimado. Para evitar esta situação duas linhas de água são usadas. Uma para apagar o incêndio no veículo enquanto que a outra arrefece os tanques com água (Fig. 3). Neste caso de estudo o utilizador irá treinar o procedimento relativo a manter a temperatura dos tanques controlada.

Uma das regras cruciais nestas situações são zonas de segurança que os bombeiros não podem violar. Tendo em atenção a segurança pessoal, o procedimento contempla que estes não se podem encontrar na parte frontal ou traseira do veículo, podem-se apenas aproximar pelas laterais. Esta situação acontece devido ao facto de que em caso de explosão existe uma maior probabilidade de os projéteis serem disparados nestas zonas. Além de que em caso de os tanques estarem posicionados de maneira a que se a válvula de segurança abrir por excesso de pressão, o gás não deverá sair pelas laterais do veículo. Este nível contempla ainda ajudas visuais no que diz respeito à temperatura e à zona de perigo (Fig. 4) para apoio à decisão do participante.

O nível urbano (Fig. 5) procura representar um acontecimento real em que o participante tem de, novamente, arrefecer o hidrogénio de um carro movido a hidrogénio, enquanto uma equipa de bombeiros virtuais extingue um fogo neste. No entanto, desta vez não existem qualquer ajudas visuais e o participante terá de recordar que procedimento realizar e as áreas de segurança a cumprir.

\section{Instrumentos}

Relativamente questionários, o SUS [16] foi usado para avaliar a usabilidade do sistema. Este utiliza uma escala de cinco pontos onde o participante responde entre o um (discordo totalmente) e o 5 (concordo totalmente). A pontuação deste questionário vai de 0 a 100, onde aplicações são no mínimo aceitáveis se tiverem pontuações superiores a 70, onde melhores aplicações pontuam entre 70 e 80. Aplicações com uma pontuação superior a 90 destacam-se positivamente das restantes [13], [20]. O ASQ [19] foi utilizado para avaliar a 


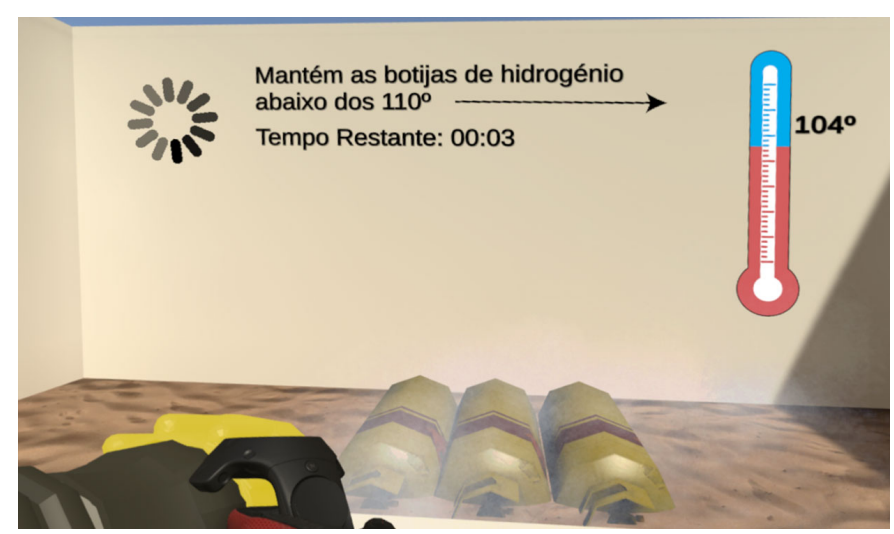

Fig. 2. Nível Tutorial com indicações sobre o procedimento relativamente ao Hidrogénio.

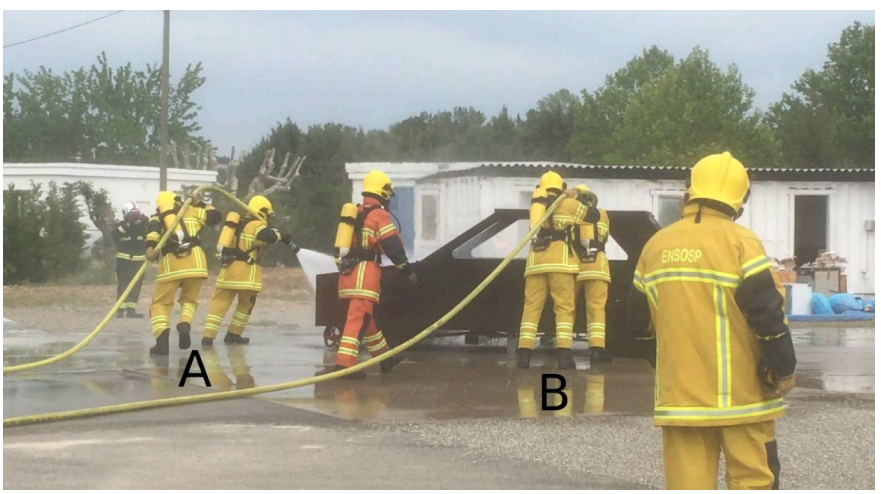

Fig. 3. Linha a para apagar o incêndio no veículo (A) enquanto outra linha arrefece os tanques com água (B).

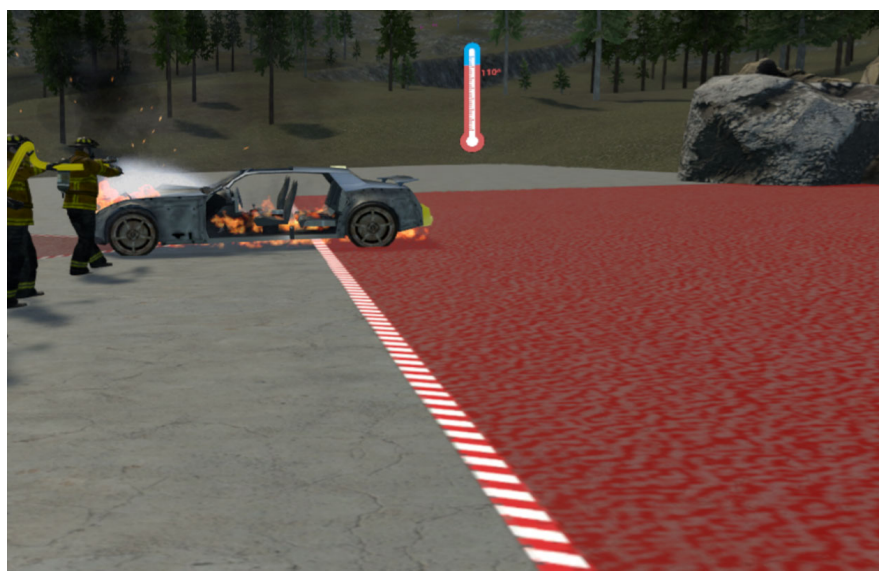

Fig. 4. Nível Treino com ajudas visuais no que diz respeito à temperatura e à zona de perigo.

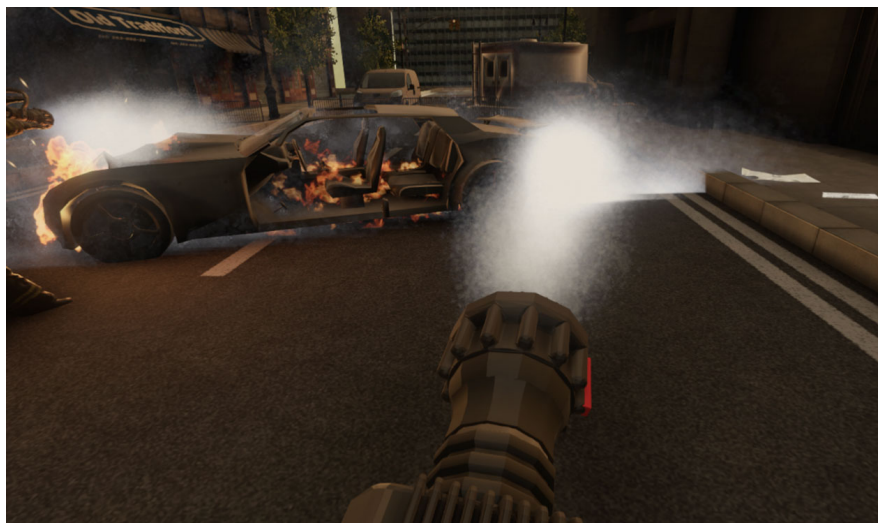

Fig. 5. Nível Urbano.

satisfação do participante com a usabilidade do sistema, como a facilidade de conclusão da tarefa, o tempo de conclusão e adequação das informações de suporte. Este é composto por seis afirmações da escala Likert, onde o participante responde entre o um (discordo totalmente) e o sete (concordo totalmente). A experiência prévia com RV, jogos e computadores foi recolhida através do questionário sociodemográfico, tal como a idade e género dos participantes. Os erros nos procedimentos foram automaticamente analisados e registados pela aplicação.

\section{E. Procedimento}

A experiência decorreu numa sala experimental com ambiente controlado, localizada no MASSIVE Virtual Reality Lab, Portugal.

Antes de ser iniciada a experiência, o participante preenche um questionário sociodemográfico básico para que a demografia possa ser obtida. Posteriormente, é informado do procedimento que irá realizar e, com a ajuda do investigador, o HMD e os auscultadores são devidamente posicionados e os comandos entregues.

Concluindo esta etapa, dá-se início à fase inicial da primeira experiência, que consiste num tutorial que dura cerca de 2 minutos. Após completar este nível, o participante preenche os questionários SUS e ASQ relativamente a esta fase.

Em seguida, o investigador volta a colocar o HMD e os auscultadores no participante, entrega-lhe os comandos e começa o nível de treino. O participante tem de arrefecer o hidrogénio durante 1 minuto. De seguida, o participante preenche novamente os questionários SUS e ASQ relativamente a esta fase.

Após a sua conclusão, o participante testa o método aprendido nos últimos dois níveis, no nível urbano. Para terminar a experiência, o participante preenche os últimos questionários SUS e ASQ que se referem a esta ultima fase.

Em média, cada participante demorou 10 a 15 minutos para completar a experiência, incluindo o preenchimento dos questionários (demográfico, SUS e ASQ). 
TABLE I

ESTATÍSTICAS DESCRITIVAS PARA PONTUAÇÃO DO SUS

\begin{tabular}{lcc}
\hline Níveis & Média & Desvio Padrão \\
\hline Tutorial & 90,05 & 11,168 \\
Treino & 89,50 & 8,317 \\
Urbano & 91,25 & 8,437 \\
\hline Total & 90,42 & 0,878 \\
\hline
\end{tabular}

TABLE II

ESTATÍSTICAS DESCRITIVAS PARA PONTUAÇÃO DO ASQ

\begin{tabular}{lcc}
\hline Níveis & Média & Desvio Padrão \\
\hline Tutorial & 6,77 & 0,353 \\
Treino & 6,44 & 0,605 \\
Urbano & 6,55 & 0,583 \\
\hline Total & 6,59 & 0,168 \\
\hline
\end{tabular}

\section{Resultados}

\section{A. Estatística descritiva}

No que diz respeito aos resultados de usabilidade medidos através do SUS (Tabela I), o nível tutorial teve um mínimo de 72,5 e um máximo de 100, dando uma média de 90,05; o nível treino teve um mínimo de 82,5 e um máximo de 100 , dando uma média de 89,50; e o nível treino teve um mínimo de 75 e um máximo de 100, uma média de 91,25; dando à aplicação um resultado de SUS de 90,42.

No que diz respeito aos resultados de satisfação, medidos através do ASQ (Tabela II), o nível tutorial teve um mínimo de 6,33 e um máximo de 7,00, dando uma média de 6,77; o nível treino teve um mínimo de 5,33 e um máximo de 6,83, dando uma média de 6,40; e o nível treino teve um mínimo de 5,17 e um máximo de 6,67, uma média de 6,55; dando à aplicação um resultado de ASQ de 6,59.

Relativamente aos erros no procedimento (Fig 6), no nível de treino como no nível urbano, $80 \%$ dos participantes mantiveram-se na zona de segurança; 50\% dos participantes não deixaram o hidrogénio aquecer além dos $110^{\circ} \mathrm{C} ; 50 \%$ dos participantes efetuaram todo o procedimento do respetivo nível corretamente. Apenas $40 \%$ dos participantes efetuaram o procedimento completo (nível treino e nível urbano) corretamente; dos $60 \%$ participantes que falharam o procedimento completo, $66,67 \%$ deles falharam nos dois níveis enquanto $33,33 \%$ falharam em apenas um dos níveis.

\section{B. Grupo que realizou procedimentos corretamente vs os que erraram}

Em regra geral, os participantes que passaram aos procedimentos tiveram uma melhor taxa de satisfação $(M=6,70$, $\mathrm{DP} \approx 0,30$ ) do que os participantes que cometeram erros durante o procedimento $(\mathrm{M}=6,49, \mathrm{DP} \approx 0,43)$. No entanto os participantes que passaram aos procedimentos tiveram uma pior pontuação de usabilidade $(\mathrm{M}=89,79 ; \mathrm{DP} \approx 7,59)$ do que os participantes que cometeram erros durante o procedimento $(\mathrm{M}=90,83 ; \mathrm{DP} \approx 8,90)$.

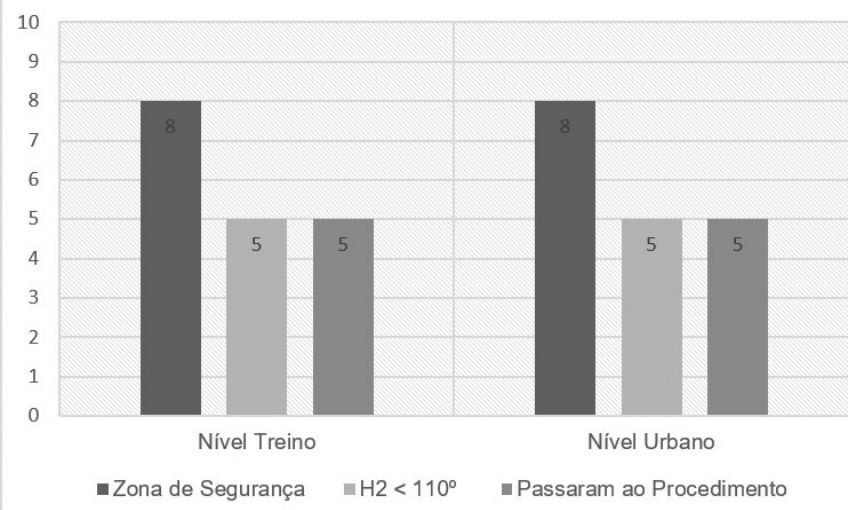

Fig. 6. Resultados dos participantes que efetuaram o procedimento corretamente, por níveis.

TABLE III

CONHECIMENTO DE RV, Jogos E COMPUTAdoRES VS REALIZAÇÃo DO PROCEDIMENTO CORRETAMENTE.

\begin{tabular}{lcccccc}
\hline & \multicolumn{2}{c}{ VR } & \multicolumn{2}{c}{ Jogos } & \multicolumn{2}{c}{ Computador } \\
\hline Procedimento & M & DP & M & DP & M & DP \\
\hline Efetuado corretamente & 6,75 & 0,500 & 6,25 & 0,957 & 6,25 & 0,500 \\
Efetuado com erros & 6,00 & 0,894 & 5,67 & 0,816 & 6,17 & 0,983 \\
\hline
\end{tabular}

De forma a verificar se estas diferenças eram significativas foi realizado um teste de Mann-Whitney U de maneira a determinar diferenças nas pontuações ASQ e SUS entre os participantes que realizaram corretamente todos os procedimentos e os que não. As pontuações de ASQ ( $U=8, z=-0,88, p=0,38)$ e SUS $(U=12, z=0, p=1)$ revelaram não ser significativamente diferentes entre os grupos.

\section{Diferenças entre níveis}

Foi realizado um teste de Kruskal-Wallis de forma a verificar se as pontuações de ASQ (Fig: 7) e SUS (Fig: 8) variam entre os níveis de Tutorial, Treino e Urbano. Os resultados indicam que não existe diferenças estatisticamente significativas no ASQ $(p=0,33)$ e SUS $(p=0.73)$ entre níveis.

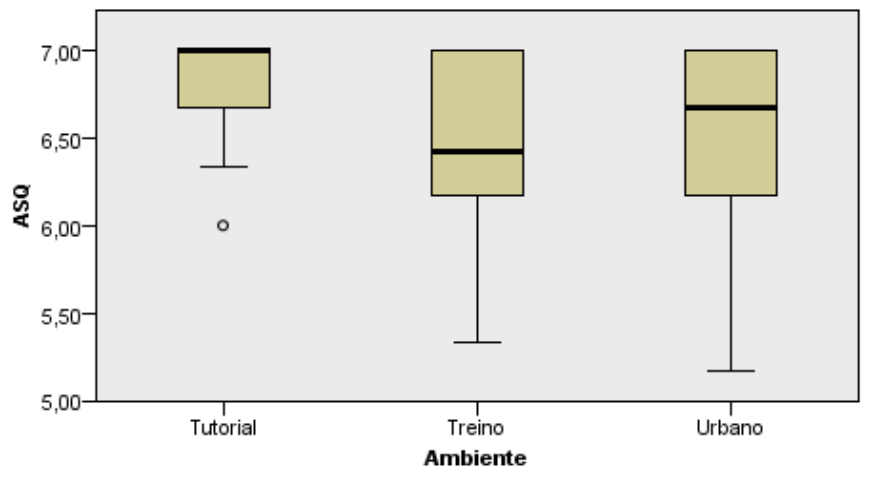

Fig. 7. Pontuações ASQ 


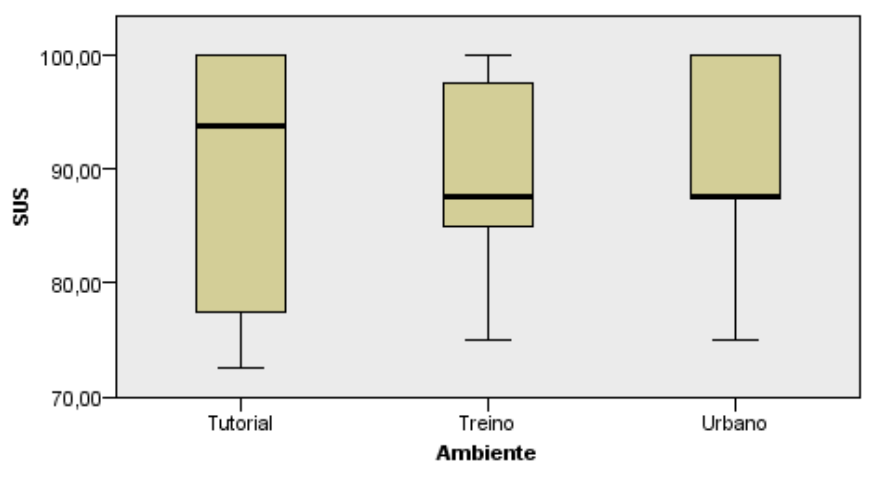

Fig. 8. Pontuações SUS

\section{Experiência prévia em VR, jogos e computador vs proced- imentos errados}

De forma a verificar se a experiência prévia dos utilizadores com a tecnologia de RV, com jogos ou em computadores afetou a capacidade de estes captarem melhor as instruções e de aplica-las de forma correta. Foram criados dois grupos através dos dados recolhidos (participantes que realizaram o total dos procedimentos corretamente $v s$ os que não) $\mathrm{O}$ teste de Mann-Whitney $U$ foi realizado de maneira a determinar se existiam diferenças na experiência prévia em RV, jogos e computador entre os grupos definidos acima. Os resultados demonstraram que não houve diferenças estatisticamente significativas na experiência prévia com $\mathrm{VR}(\mathrm{U}=6 ; \mathrm{z}=-1,389$, $\mathrm{p}=0,165)$, jogos $(\mathrm{U}=7,5 ; \mathrm{z}=-1,016, \mathrm{p}=0,309)$ e computador $(\mathrm{U}=12 ; \mathrm{z}=0, \mathrm{p}=1)$.

\section{DISCUSSÃo}

Este estudo teve como foco a usabilidade do sistema, a satisfação do utilizador e os erros no procedimento de treino. No que diz respeito à usabilidade do sistema, medida com recurso ao SUS, todos os três níveis deste estudo foram superiores a 89,5 e uma média geral da usabilidade da aplicação de 90,42 ( $\mathrm{DP} \approx 0,878$ ), podemos dizer que que a aplicação deste estudo não só se encontra no quarto quartil, como pode ser considerada excelente no que diz respeito à usabilidade [13].

Em termos de satisfação do utilizador, considerando que o valor máximo do ASQ é 7 [19] e que esta aplicação atingiu uma média de 6,59, podemos verificar que este se encontra a $94,14 \%$ da escala. Como tal, consideramos que os participantes ficaram muito satisfeitos com esta aplicação. Estes resultados positivos vão de encontro aos estudos de Tuli Sulbaran e Nelson C. Baker [3] e de Shana Smith e Emily Ericson [4] onde estes afirmam que os participantes têm preferência por este tipo de aprendizagem.

Quando comparados os grupos que passaram ao procedimento e os que não passaram com os dados sociodemográficos referentes à experiência prévia com VR, jogos e computadores (Tabela III) foi percetível que, apesar de o grupo que falhou o procedimento ter registado uma pontuação ligeiramente mais baixa no que diz respeito à experiência de VR, jogos e computadores, as diferenças não foram estatisticamente significativas.

Tendo em conta as observações feitas durante o procedimento dos participantes que cometeram erros, alguns perderam uma porção importante do tempo a apanhar o bocal da mangueira e a teletransportar-se até ao carro, o que implicou um aquecimento do hidrogénio superior a $110^{\circ} \mathrm{C}$ ainda antes do participante começar a arrefecê-lo. Esta limitação advém da possível dificuldade de alguns utilizadores em recordar como interagir com a mangueira através dos comandos. Alguns participantes demonstraram ainda alguma perda de foco enquanto arrefeciam as botijas de hidrogénio, cujo pode ser devido ao facto de terem de manter a temperatura dos tanques controlada ao dirigir a água para estes. A perda de foco pode ainda estar relacionada com o facto de os utilizadores não receberem feedback da ação que estão a efetuar (para além de uma indicação visual da temperatura atual dos tanques no nível de treino). Além deste ponto, o facto de manterem a direção da água para um tanque que à primeira vista parece inofensivo, enquanto que o resto do veículo se encontra em chamas, pode ser contra intuitivo. Esta situação possivelmente levou os utilizadores a começar a ajudar a equipa de bombeiros virtuais a apagar o incêndio resultando num aumento de temperatura dos tanques para além do ponto crítico de $110^{\circ} \mathrm{C}$.

Como o nível urbano não continha ajudas visuais, houve participantes que se encontravam ligeiramente dentro da área de perigo.

Os resultados indicaram que não houve diferenças significativas entre os níveis. Isto indica que o sistema ofereceu diferentes contextos da simulação (tutorial, com ajudas visuais, etc,.) sem prejudicar a satisfação e usabilidade.

O nível urbano, não tendo ajudas visuais, está considerado como o nível de procedimento mais difícil e, como tal, deveria ter uma maior percentagem de erros cometidos pelos participantes. No entanto, não existiu um aumento de erros cometidos no nível urbano quando comparado com o nível de treino. Colocamos em hipótese que isto se deveu ao nível de tutorial, que, quem entendeu as instruções do procedimento, conseguiu aplicá-las com sucesso ao longo da experiência. Em parte, o elevado número de participantes que cometeram erros nos procedimentos pode ser devido ao curto tutorial. Este possivelmente não foi suficiente para levar certos participantes a fixar a informação necessária de forma a aplicar adequadamente os procedimentos.

\section{CONClus Ão}

O principal objetivo deste estudo era tentar perceber, a nível exploratório, se seria usável e satisfatório a utilização de uma aplicação de treino e certificação de bombeiros em RV com o intuito de aprendizagem e aprimoramento dos conhecimentos perante situações de perigo relacionadas com libertação de hidrogénio em veículos ligeiros.

Este estudo contribui para uma melhor compreensão do potencial da tecnologia de RV para apoiar e melhorar o treino e certificação de bombeiros, mais especificamente situações que envolviam a libertação de hidrogénio em automóveis. 
Os resultados demonstraram que os participantes indicaram como muito positiva esta aplicação em termos de usabilidade e satisfação.

\section{LIMITAÇÕES E TRABALHO FUTURO}

Uma das limitações deste estudo é o pequeno tamanho da amostra. No entanto, a realização deste estudo irá permitir aprimorar a aplicação desenvolvida e desenvolver novos módulos de formação tendo em conta os resultados obtidos. Nesse seguimento, estão previstos estudos de maneira a estender a mais participantes. Relativamente ainda à amostra, esta foi composta por pessoas sem formação nesta profissão e, como tal, seria necessário testar esta aplicação com bombeiros profissionais ou voluntários e comparar com situações de treino reais.

Espera-se também estender esta aplicação baseada em RV para incorporar outras atividades específicas do treino e certificação de bombeiros. Ainda relacionado com a libertação de hidrogénio, espera-se adicionar a esta aplicação em situações que envolvem não só automóveis mas também autocarros, válvulas, entre outros. Para a mesma situação é possível que bombeiros tenham de desempenhar tarefas diferentes, sendo igualmente importante adicionar a possibilidade de simular diferentes papeis nestas simulações e até mesmo ser possível realizar a simulação com vários utilizadores interagindo entre si. Como exemplo temos a opção dos utilizadores poderem estar não só na linha que arrefece os tanques com água, mas também de poderem realizar o procedimento na linha para apagar o incêndio (Fig. 3). Posteriormente pretendemos efetuar outro tipo de estudos, nomeadamente o sentimento de Presença e respetivas sub escalas.

\section{AgRADECIMENTOS}

Este trabalho foi financiado por Fundos FEDER através do Programa Operacional Competitividade e Internacionalização - COMPETE 2020 e por Fundos Nacionais através da FCT Fundação para a Ciência e a Tecnologia no âmbito do projeto POCI-01-0145-FEDER-028618.

\section{REFERÊNCIAS}

[1] S. P. Smith and D. Trenholme, "Rapid prototyping a virtual fire drill environment using computer game technology," Fire safety journal, vol. 44, no. 4, pp. 559-569, 2009.

[2] J. Dugdale, B. Pavard, N. Pallamin, M. El Jed, and C. L. Maugan, "Emergency fire incident training in a virtual world," Proceedings ISCRAM, vol. 167, 2004.

[3] T. Sulbaran and N. C. Baker, "Enhancing engineering education through distributed virtual reality," in 30th Annual Frontiers in Education Conference. Building on A Century of Progress in Engineering Education. Conference Proceedings (IEEE Cat. No. OOCH37135), vol. 2. IEEE, 2000, pp. S1D-13.

[4] S. Smith and E. Ericson, "Using immersive game-based virtual reality to teach fire-safety skills to children," Virtual reality, vol. 13, no. 2, pp. 87-99, 2009.

[5] D. L. Tate, L. Sibert, and T. King, "Virtual environments for shipboard firefighting training," in Proceedings of IEEE 1997 Annual International Symposium on Virtual Reality. IEEE, 1997, pp. 61-68.

[6] L. Gamberini, P. Cottone, A. Spagnolli, D. Varotto, and G. Mantovani, "Responding to a fire emergency in a virtual environment: different patterns of action for different situations," Ergonomics, vol. 46, no. 8, pp. 842-858, 2003.
[7] T. U. St Julien and C. D. Shaw, "Firefighter command training virtual environment," in Proceedings of the 2003 conference on Diversity in computing. ACM, 2003, pp. 30-33.

[8] M. Cha, J. Lee, B. Choi, H. Lee, and S. Han, "A data-driven visual simulation of fire phenomena," in SIGGRAPH'09: Posters. ACM, 2009, p. 74 .

[9] P. Backlund, H. Engstrom, C. Hammar, M. Johannesson, and M. Lebram, "Sidh-a game based firefighter training simulation," in 2007 11th International Conference Information Visualization (IV'07). IEEE, 2007, pp. 899-907.

[10] J. Lee, M. Cha, B. Choi, and T. Kim, "A team-based firefighter training platform using the virtual environment," in Proceedings of the 9th ACM SIGGRAPH Conference on Virtual-Reality Continuum and its Applications in Industry. ACM, 2010, pp. 299-302.

[11] A. Ren, C. Chen, J. Shi, and L. Zou, "Application of virtual reality technology to evacuation simulation in fire disaster." in CGVR. Citeseer, 2006, pp. 15-21.

[12] E. Perdigau, P. Torguet, C. Sanza, and J.-P. Jessel, "A distributed virtual storytelling system for firefighters training," in International Conference on Virtual Storytelling. Springer, 2003, pp. 227-230.

[13] A. Bangor, P. T. Kortum, and J. T. Miller, "An empirical evaluation of the system usability scale," Intl. Journal of Human-Computer Interaction, vol. 24, no. 6, pp. 574-594, 2008.

[14] D. A. Bowman and R. P. McMahan, "Virtual reality: how much immersion is enough?" Computer, vol. 40, no. 7, pp. 36-43, 2007.

[15] J. Kirakowski and M. Corbett, "Sumi: The software usability measurement inventory," British journal of educational technology, vol. 24, no. 3 , pp. 210-212, 1993.

[16] J. Brooke et al., "Sus-a quick and dirty usability scale," Usability evaluation in industry, vol. 189, no. 194, pp. 4-7, 1996.

[17] A. Rich and M. McGee, "Expected usability magnitude estimation," in Proceedings of the Human Factors and Ergonomics Society Annual Meeting, vol. 48, no. 5. SAGE Publications Sage CA: Los Angeles, CA, 2004, pp. 912-916.

[18] H. Coelho, M. Melo, L. Barbosa, J. Martins, M. S. Teixeira, and M. Bessa, "Authoring tools for creating 360 multisensory videos-evaluation of different interfaces," Expert Systems, p. e12418, 2019.

[19] J. R. Lewis, "Ibm computer usability satisfaction questionnaires: psychometric evaluation and instructions for use," International Journal of Human-Computer Interaction, vol. 7, no. 1, pp. 57-78, 1995.

[20] J. Brooke, "Sus: a retrospective," Journal of usability studies, vol. 8, no. 2, pp. 29-40, 2013. 